International Journal of Food Science, Nutrition and Dietetics (IJFS)

ISSN 2326-3350

\title{
Role of Dietary Soybean Genistein in Osteoporosis Prevention
}

Masayoshi Yamaguchi ${ }^{1 *}$, Ying Hua Gao-Balch ${ }^{2}$

Review Article

${ }^{1 *}$ Division of Diabetes, Endocrinology and Metabolism, Department of Medicine, Baylor College of Medicine, Houston, TX 77030, USA.

${ }^{2}$ Department of Human Science, University of Arkansas at Pine Bluff, 1200 North University Drive, Pine Bluff, Arkansas 71601, USA.

\section{Abstract}

Bone homeostasis is regulated through osteoclasts and osteoblasts. Osteoporosis, which is induced with its accompanying decrease in bone mass with increasing age, is widely recognized as a major public heath problem. Bone loss may be due to decreased osteoblastic bone formation and increased osteoclastic bone resorption. There is growing evidence that nutritional and food factors may play a part in the prevention of bone loss with aging and have been to be worthy of notice in the prevention of osteoporsis. Genistein, which is contained in soybeans, has been shown to have a stimulative effect on osteoblastic bone formation and a suppressive effect on osteoclastic bone resorption, thereby increasing bone mass. This factor has a regulative effect on protein synthesis and gene expression, which are related to bone formation in osteoblastic cells and bone resorption in osteoclastic cells. Oral administration of genistein has been shown to prevent on bone loss in ovariectomized rats, an animal model for osteoporosis, indicating a role in the prevention of osteoporosis. Intake of dietary soybean genistein has been shown to have a preventive effect on osteoporosis in human subjects, suggesting a role in the prevention of bone loss. This review discusses a role of dietary soybean genistein in osteoporosis prevention.

Keywords: Bone, Osteoporosis, Genistein, Osteoblastic bone formation, Osteoclastic bone resorption

\section{*Corresponding Author:}

Masayoshi Yamaguchi,

Division of Diabetes, Endocrinology and Metabolism, Department of Medicine, Baylor College of Medicine,

One Baylor Plaza, Houston, TX 77030, USA.

Tel: 713-798-6686, Fax: 713-798-8764.

E-mail: yamamasa1155@yahoo.co.jp

Received: February 19, 2013

Accepted: February 25, 2013

Published: February 26, 2013

Citation: Yamaguchi M, Y H Gao-Balch(2013) M Role of Dietary Soybean Genistein in Osteoporosis Prevention. Int J Food Sci Nutr Diet. 2(2 ), 27-34. doi: http://dx.doi.org/10.19070/2326-3350-130006

Copyright: Yamaguchi $\mathbf{M}^{\mathscr{0}}$ 2013. This is an open-access article distributed under the terms of the Creative Commons Attribution License, which permits unrestricted use, distribution and reproduction in any medium, provided the original author and source are credited.

\section{Introduction}

Bone homeostasis is skillfully regulated through osteoclasts and osteoblasts. Aging induces a reduction in bone mass by decrease in osteoblactic bone formation and increase in osteoclastic bone resorption. Osteoporosis with its accompanying decrease in bone mass is widely recognized as a major public health problem. The most dramatic expression of the disease is represented by fractures of the proximately femur, and the number of which increases as the population ages [1]-[3]. Malnutrition or undernutrition is often observed in the elderly, and it appears to be more intense in patients with hip fracture than in the general aging population [4]. Deficiency in both micronutrients and macronutrients appears to be strongly implicated in the pathogenesis and the consequences of hip fracture in the osteoporotic elderly.
There is growing evidence that nutritional factors have a role in the prevention of bone loss with increasing age [5]-[7]. Food life may help to prevent bone loss with aging. Chemical compounds in food and plants, which regulate bone homeostasis, have been to be notice. Recent studies have been shown that nutritional and functional food factors, which are present in fruit and vegetables, stimulate osteoblastic bone formation and suppresses osteoclastic bone resorption in osteoporosis animal models and human subjects [5]-[7], thereby increasing bone mass. Supplemental intake of ingredients plays a role in maintaining of bone health and in the prevention of bone loss.

This review introduces recent findings concerning the role of soybean genistein in the regulation of bone homeostasis and in the prevention of osteoporosis in animal models and human subjects.

\section{Genistein stimulates osteoblastic bone formation}

Bone cells in bone homeostasis. Bone homeostasis is regulated through the functions of osteoblasts and osteoclasts, which are major cells in bone tissues [8]-[14]. Osteoblasts, which are differentiated from bone marrow messenchymal stem cells, stimulate bone formation and calcification, while osteoclasts, which develop from hematopoietic progenitors, promote bone resorption. Osteoclasts are recruited to the site and excavate the calcified matrix. In the physiologic process of bone turnover, a resorptive stimulus firstly triggers recruitment of osteoclasts to a site on the bone surface. After the resorbed lacunar pit is filled with new osteoid, osteoblasts become flatter and less active, with the final newly remodeled bone surface lined by flat lining cells. Remodeling of cancellous bone begins with the retraction of lining cells that cover the bone surface [13].

Bone acts as major storage site for growth factors, which are produced by osteoblasts, diffuse into newly deposited osteoid and 
are stored in the bone matrix including isulin-like growth factors (IGF- I and II), transforming growth factor- $\beta 1$ (TGF- $\beta 1$ ), platelet-derived growth factor (PDGF), or bone morphologic proteins (BMPs) [12]-[14]. These bone-derived factors, which can be liberated during subsequent periods of bone resorption, act in an autocrine, paracrine, or delayed paracrine fashion in the local microenvironment of the bone surface. The process of bone remodeling to make bone is unique among organs and tissues, and it is complexity with respect to interactions along the remodeling sequence by systemic influences (hormones), stress action on trabecular and cortical systems, growth factors and cytokines produced by the bone cells which act locally on their own cell types and on the other bone cell types, or factors that come from nearby cells present in the bone marrow tissues. Functional food factors may have a regulatory effect on the process of bone remodeling.

Genistein has an anabolic effect in bone tissues. Isoflavones (including daidzin, daidzein, genistein, and genistein) are contained in soybeans with relatively high concentrations. Daidzin or genistin is hydrolyzed to daidzein or genistein by $\beta$-glucosidase in the gastrointestinal system, respectively. Genistein has been found to have a strong inhibitory effect on protein tyrosine kinase and it can cause cell cycle arrest and apotosis in leukemic cells [15]. This cellular mechanism of genistein may be important in the prevention of cancers. Yamaguchi et al.found that genistein or daidzein has been demonstrated to have a direct anabolic effect on bone metabolism in vitro [16], suggesting a role in the prevention of osteoporosis. This was the first time finding. Genistein as a food factor is usefulness in the prevention and treetment of osteoporosis.

The anabolic effects of genistein on bone metabolism have been demonstrated in tissue culture using the femoral metaphyseal (trabecular bone) tissues obtained from elderly female rats in vitro [16],[17]. Bone tissues were cultured for 24 hours in a Dulbecco's modified Eagle's medium (high glucose, 4.5\%) with bovine serum albumin (serum free) containing either vehicle or genistein. The presence of genistein $\left(10^{-6}\right.$ or $\left.10^{-5} \mathrm{M}\right)$ caused a significant increase in bone components (cal cium content, alkaline phosphatase activity, and DNA content) in rat femoral metaphyseal tissues [16],[17]. The effect of genistein in increasing bone components is equal to the stimulatory effects of $17 \beta$-estradiol, which is important anabolic hormone in bone metabolism. Antiestrogen agent tamoxifen inhibited the genistein-induced increase in bone components, although tamoxifen itself had no effect on bone components [16]. The anabolic effects of genistein on bone metabolism may be partly mediated through etrogen-like action. Presumably, genistein binds to the receptor of estrogen in osteoblastic cells in which the receptor is localized [18].

The effects of genistein or genistin on bone components have been compared in an in vitro culture system [16]. Culture with genistein $\left(10^{-8}-10^{-5} \mathrm{M}\right)$ or genistin $\left(10^{-7}-10^{-5} \mathrm{M}\right)$ increased alkaline phosphatase activity and deoxyribonucleic acid (DNA) and calcium contents in the femoral-metaphyseal tissues obtained from elderly female rats [16]. The effect of genistein was greater than that of genistin. The effects of genistein or genistin in increasing bone components were blocked in the presence of cycloheximide, an inhibitor of protein synthesis in the translational process, suggesting that the anabolic effects of the isoflavones are partly resulted from newly synthesized protein components.

The anabolic effects of daizein on the femoral-diaphyseal (cortical bone) tissues obtained from elderly female rats in vitro have also been shown [19]. The presence of daidzein $\left(10^{-6}\right.$ or $\left.10^{-5} \mathrm{M}\right)$ in culture medium caused a significant increase in bone components. The effect of daizein was equal to that of genistein $\left(10^{-6}\right.$ or $10^{-5}$ M). The combination of daidzein and genistein did not have an additive effect [19]. In addition, the stimulating effect of daidzein on bone components was completely suppressed in the presence of cycloheximide [19], suggesting that its effect is resulted from protein synthsis.

The effects of phosphogenistein and phosphodaidzein, which are phosphorylated for the hydroxyl group at the 7-position of genistein and daidzein, on bone components has been compared to the effect of genistein or daizen [20]. Phosphogenistein or phosphodaidzein increased bone components in tissue culture in vitro. Phosphoisoflavones with a lower concentration of 10-6 M, at which genistein and daidzein have anabolic effects on bone components, did not have an effect [20].

The effects of various polyphenols found in food and plants on bone metabolism have also been compared [21]. Glycitein, resveratol, quercetin, catechin, and (-)-epigallocatechin gallate did not have an anabolic effect on bone calcification in vitro [21]. Polyphenols did not have a direct stimulative effect on bone calcification in vitro. Among various polyphenol, genistein has a unique anabolic effect on bone.

As the mentioned above, genistein is a 4, 5, 7-trihydroxyisoflavone, and daidzein is a 4',7-dihydroxyisoflavone. Glycitein is a 4', 7-dihydroxy-6-methoxyflavone, while quercetin is a 3, 3', 4', 5, 7-pontahydeoxyflavone; neither has any effect of genistein and daidzein on bone calcification is weakened by the phosphorylation of the hydroxy-group at their-7-position [21]. The hydroxygroups at the 5- and 7-positions of the isoflavone genistein may be necessary for its anabolic effect on bone formation, suggesting a chemical structure-activity relationship. Presumably, the chemical form of dihydroxyisoflavone is important in revealing its anabolic effects on bone. Genistein and daidzein may have bone effect that operates via the same mechanism.

Genistein stimulates osteoblastogenesis. Osteoblasts play an essential role in the stimulation of bone formation and calcification. The anabolic effect of isoflavones on osteoblastic cells in vitro has been demonstrated [22-24]. Osteoblastic MC3T3-E1 cells were cultured for 48 hours in a serum-free modified Eagle's medium containing genistein or daidzein. The presence of genistein $\left(10^{-6}\right.$ or $\left.10^{-5} \mathrm{M}\right)$ or daidzein $\left(10^{-6}\right.$ or $\left.10^{-5} \mathrm{M}\right)$ caused a significant increase in protein content, alkaline phosphatase activity and DNA content in the cells [22]. The ability of genistein or daidzein to increase biochemical components in the cells was not seen in the presence of an inhibitor of protein synthesis [22], suggesting that the effects of isoflavones result from newly synthesized protein components. The anabolic effects of genistein in osteoblastic cells are not distinguishable from those of daidzein [22,23].

The effects of $17 \beta$-estradiol in increasing protein content and alkaline phosphatase activity in osteoblastic cells were not enhanced in the presence of genistein [22]. Cellular protein content was additively increased with $17 \beta$-estradiol and daidzein, but their effects on alkaline phosphatase activity were not additively. The receptors of estrogen are found in osteoblastic cells. Genistein has been shown to bind to estrogen receptor $\beta$ in osteoblastic cells [18], and daidzein cannot bind to the receptor [18]. It is speculated that genistein may bind to estrogen receptor $\beta$ in osteoblastic cells, although the nuclear localization of genistein in these cells has poorly understood. 
DNA content in osteoblastic cells was increased in the presence of genistein or daizein, suggesting that isoflavones stimulate cell proliferation [22,23]. Isoflavones increase alkaline phosphatase activity, which is a marker enzyme in the differentiation of osteoblastic cells. Genistein and daidzein may have the stimulatory effects on the proliferation and differentiation of osteoblastic MC3T3-E1 cells.

The cellular mechanism by which isoflavones stimulate osteoblastic bone formation has been studied in relation to protein synthesis [24]. The presence of genistein or daidzein stimulated protein synthesis in osteoblastic MC3T3-E1 cells in vitro [24]. The addition of genistein or daidzein into the reaction mixture of protein synthesis using the cell homogenate from osteoblastic cells cultured without isoflavone caused a significant increase in protein synthesis in vitro [24]. This increase was suppressed in the presence of an inhibitor of protein synthesis, indicating that genistein or daidzein can directly stimulate protein synthesis in vitro. Moreover, genistein or daidzein has been shown to increase $[3 \mathrm{H}]$-leucyl-tRNA synthetase activity in the cytosol fraction of osteoblastic cell homogenate [24]. Genistein had a greater effect than daidzein [24]. Isoflavone can directly activate leucyl-tRNA synthetase, a rate-limiting enzyme in the translational process of protein synthesis.

The possibility that genistein or daidzein acts on the translational process in osteoblastic MC3T3-E1 cells cannot exclude, however, since the stimulating effects of the isoflavones on protein synthesis in osteoblastic cells are suppressed by actinomycin D, an inhibitor of transcription activity [24]. Genistein has been shown to bind to estrogen receptors in osteoblastic cells [13], although daidzein cannot bind to estrogen receptors [18]. Moreover, it is possible that genistein or daidzein can bind to other transcription factors, which differ from estrogen receptors, in osteoblastic MC3T3-E1 cells.

The estrogenic compounds of $17 \beta$-estradiol and genistein mediate very different actions on osteoblastic cells [25]. While $17 \beta$-estradiol may stimulate bone anabolism, in part, by antagonizing $\mathrm{TNF} \alpha$-induced $\mathrm{NF}-x \mathrm{~B}$ activation, genistein did not only fails to prevent the cytokin-induced $\mathrm{NF}-x \mathrm{~B}$ activation, but directly promotes NF- $x \mathrm{~B}$ activation in MC3T3 cells [25]. These observations suggest an important mechanistic difference in the mechanisms by which $17 \beta$-estradiol and genistein promote osteoblast differentiation [25]. Whether genistein has an effect on various protein kinases and protein phosphatases that are related to osteoblastic cell proliferation and nuclear transcription activity remains to be elucidated.

Molecular mechanism by which genistein stimulates osteoblastogenesis. The cellular and molecular mechanism by which genistein enhances osteoblastogenesis has been examined [26,27]. Culture with genistein $\left(10^{-5} \mathrm{M}\right)$ has been found to have a stimulative effect on the mineralization in osteoblastic cells in vitro [26]. Alkaline phosphatase is an enzyme marker of osteoblasts and the enzyme participates in bone mineralization [27]. $\alpha 1$ (I) collagen is a matrix protein that is related to bone formation and mineralization in osteoblast lineage cells [28]. Osteocalcin is a bone matrix protein containing $\gamma^{2}$-carboxyglutamin acid, which is synthesized in osteoblasts and is protein involved in mineralization [28]. The expression of alkaline phosphatase, $\alpha 1$ (I) collagen, and osteocalcin mRNAs in osteoblastic cells was markedly enhanced after culture with genistein $\left(10^{-6}\right.$ or $\left.10^{-5} \mathrm{M}\right)$ as compared to the effect of each factor [26].

The effect of genistein $\left(10^{-5} \mathrm{M}\right)$ on the gene expression in osteoblastic cells was completely depressed in the presence of cycloheximide, an inhibitor of protein synthesis, and 5,6-dichlorobenzimidazone-1- $\beta$-D-ribofuranoside (DRB), an inhibitor of transcriptional activity [26]. This suggests that genistein stimulates transcriptional process in osteoblastic cells. Genistein may have a stimulative effect on protein synthesis at the transcriptional process. Genistein $\left(10^{-6}\right.$ or $\left.10^{-5} \mathrm{M}\right)$ was found to increase protein content in osteoblastic MC3T3-E1 cells in vitro [26]. This effect of genistein may involve in the activation of aminoacyl-tRNA synthase, a rate-limiting enzyme in protein synthesis. Genistein may stimulate both transcriptional and translational activities in osteoblastic cells. It is speculated that genistein has an effect on the binding of transcriptional factor(s), which is synthesized through translational process, to the nuclear DNA in osteoblastic cells.

Genistein $\left(10^{-6} \mathrm{M}\right)$ has been shown to result in an increase in cell proliferation and stimulated osteoblastic maturation as assessed by culture duration-dependent increments in alkaline phosphatase activity, calcium deposition into extracellular matrix and Runx2/ Cbfa1 gene expression in primary mouse bone marrow-derived mesenchymal stem cells (BMSCs) cultures [29]. Genistein also resulted in an increase in nitric oxide (NO) synthase activity, NO formation, and cyclic guanosine 3', 5'-monophosphate production in BM-SCs cultures [29]. These effects of genistein were mimicked by $17 \beta$-estradiol [29].

Whether genistein and daidzein regulate target gene transcription through cyclic adenosine 3', 5'-monophosphate (cAMP) regulatory element (CRE) in human osteoblastic cell line MG-63 cells has been shown [30]. It was found that $17 \beta$-estradiol, genistein and daidzein suppressed the transcriptional activity of CRE-luciferase reporter gene in MG-63 cells [30]. 17 $\beta$-estradiol and genistein but not daidzein inhibited the cAMP analogue 8-Br cAMP-induced transcription of CRE reporter gene [30]. Both genistein and $17 \beta$-estradiol inhibited basal and cAMP-induced mRNA levels of endogenous estrogen responsive genes containing CRE/CRElike elements in their promoter regions, including interleukin (IL) 8 and serum- and glucocorticoid-inducible kinase 1 [30]. These findings indicate that genistein and daidzein may modulate bone remodeling through ERs by regulating target gene expression through the CRE motifs.

\section{Genistein suppresses osteoclastic bone resorp- tion}

Genistein suppresses bone resorption in bone tissues. Parathyroid hormone (PTH), prostaglandin E2 (PGE2), and lipopolysaccharide (LPS) have a stimulative effect on bone resorption in in vitro culture system [31-33]. Culture with PTH, PGE2, or LPS clearly stimulated bone resorption in the femoral-metaphyseal tissues cultured for 48 hours, when bone resorption was estimated with a decrease in bone calcium content [34]. The effects of boneresorbing factors were completely suppressed in the presence of genistein, indicating an inhibition of bone resorption [34].

PTH and PGE2 caused a remarkable increase in glucose consumption and lactic acid production by bone tissues [34]. The production of lactic acid by bone tissues may be partly related to the augmentation of glucose consumption. Presumably, PTHand PGE2-stimulated lactic acid productions in bone tissues 
hasten a decrease in bone calcium content. Genistein completely depressed the PTH- or PGE2-induced increase in both glucose consumption and lactic acid production in bone tissues [34]. The inhibitory effects of genistein on bone resorption may be partly related to the prevention of lactic acid production by bone tissues.

Thus, genistein has a stimulative effect on both bone formation and mineralization in tissue culture in vitro, and it has also been found to have a suppressive effect on bone resorption in tissue culture. Genistein may have an important role in the preservation of bone mass.

Genistein suppresses osteoclastogenesis. Osteoclasts, bone-resorbing cells, are formed from bone marrow stem cells $[10,11$, ,35]. Osteoclast-like cell formation is estimated using staining for tartrate-resistant acid phosphatase (TRACP), a marker enzyme of osteoclasts [35]. Mouse bone marrow cells were cultured for 7 days in a medium containing a bone-resorbing agent [PTH, PGE2, 1, 25-dihydroxyvitamin D3 (VD3), or LPS] with an effective concentration [36]. Culture with PTH, PGE2, VD3, or LPS induced a remarkable increase in osteoclast-like multinucleated cells. These increases were depressed in the presence of genistein [36]. The suppressive effects of genistein (10-5 M) were equal to the effects of other anti-bone-resorbing agents (calcitonin, $17 \beta$-estradiol, and zinc sulfate) on osteoclast-like cell formation in mouse marrow culture [36].

Genistein has been shown to have a potent suppressive effect at the later stage of differentiation of bone marrow cells [36]. The suppressive effect of genistein on osteoclast-like MNC formation in mouse bone marrow culture was greater than that of daidzein. The suppressive effect of genistein on osteoclast-like MNC formation induced by PTH or PGE2 may be partly based on the blocking of the pathway of cyclic AMP signaling, but not that by protein kinase $\mathrm{C}$ signaling, at the differentiation stage of bone marrow cells [36]. In addition, if genistein can inhibit tyrosine kinase [15], the action of isoflavone on osteoclastic cell formation may be partly related to its inhibitory effect on the kinase. The cellular mechanism by which genistein inhibits osteoclast-like cell formation from marrow cells may also be involved in cyclic AMP signaling.

Interleukin-6 (IL-6) has been known to stimulate osteoclastogenesis and osteoprotegerin (OPG) depress binding of RANKL to RANK, which is receptor protein of RANKL [10]. These factors are produced in osteoblastic cells [10]. OPG mRNA expression has been found to increase in osteoblastic cells treated with $17 \beta$-estradiol, genistein, or daidzein [37]. The release of IL-6 in the cells was suppressed, while OPG production was enhanced by isoflavone treatments [37]. After adding the estrogen receptorbinding blocker, ICI-182780, the effects of isoflavones on OPG and IL-6 production disappeared [37] Thus, the inhibition by dietary isoflavones of IL-6 production and the stimulation of OPG appear to be mediated, at least in part, via a genomic pathway operating through estrogen receptors and gene expression mechanisms [37]. The stimulation of OPG mRNA levels by genistein was not affected by the protein synthesis inhibitor, cycloheximide and was shown to be due to enhancement of OPG gene transcription [38]. Thus, genistein may partly suppress bone resorption and bone loss by enhanced osteoblastic production of OPG.

RANKL or tumor necrosis factor- $\alpha(\mathrm{TNF}-\alpha)$ have been shown to stimulate osteoclastic differentiation using RAW264.7, a murine monocytic cell line. The direct effects of estrogen on osteoclast precursor differentiation has been shown that, in addition to effecting osteoblasts, estrogen may protect bone by reducing osteoclast production [39]. Both genistein and estrogen has been found to inhibit RANKL-induced IkappaB degradation and NF-kappaB $(\mathrm{NF}-x \mathrm{~B})$ nuclear localization, which is a intracellular signaling factor of RANKL, with the same relative potency as seen in proliferation and differentiation assays [39]. Genistein has been shown to depress TNF- $\alpha$-induced c-fos and NFATc1 expressions in an estrogen receptor-dependent manner and also inhibit NFATc1 nuclear translocation [40]. This finding suggests that phytoestrogens suppress TNF- $\alpha$-induced osteoclastogenesis via inhibition of c-fos-dependent NFATc1 expression [40].

Action of genistein in mature osteoclasts. Genistein has been found to induce cell death (apoptosis) of osteoclasts isolated from rat femoral tissues $[41,42]$. Calcitonin has been known to inhibit osteoclast activity [43]. This hormonal effect may be mediated through the two pathways of cyclic AMP and $\mathrm{Ca}^{2+}$ signalings [49]. The addition of dibucaine, an antagonist of calmodulin, or staurosporine, an inhibitor of pretin kinase $\mathrm{C}$, completely prevented the decrease in osteoclasts induced by calcitonin, DcAMP, or calcium chloride [41]. The effect of genistein in decreasing osteoclasts was clearly suppressed by these inhibitors, suggesting that the effect of isoflavones on mature osteoclasts is partly mediated through the pathway of $\mathrm{Ca}^{2+}$ signaling.

$\mathrm{A} \mathrm{Ca}^{2+}$ ionophore (A23187) caused a remarkable decrease in the number of osteoclasts, indicating that the entry of $\mathrm{Ca}^{2+}$ in the cells induces death of osteoclasts [44]. The number of osteoclasts was decreased, when were cultured in the presene of genistein or calcium chloride. $\mathrm{Ca}^{2+}$ can activate endonuclease and the metal induces apoptosis in cells [45]. The effect of A23187 in decreasing osteoclasts may be based on $\mathrm{Ca}^{2+}$-activated DNA fragmentation, which induces apoptosis. Genistein has been shown to induce apoptosis in immature human thymocytes by inhibiting torosisomerase II [46].

Genistein may stimulate apoptosis of osteoclasts, and its mechanism is partly related to the pathway of $\mathrm{Ca}^{2+}$ signaling. It is possible that genistein stimulates $\mathrm{Ca}^{2+}$ entry into osteoclasts, since it has been reported that the isoflavone can directly open a chloride channel in human cystic fibrosis transmembranes [41]. In addition, daidzein has also been shown to suppress the number of mature osteoclasts [41], although daidzein did not have a greater suppressive effect than genistein. The effect of daidzein was abolished in the presence of dibucaine or staurosporine, supporting a partial involvement in the $\mathrm{Ca}^{2+}$-signaling mechanism [41].

Culture with genistein has been found to cause an inhibition of protein kinase activity and an appreciable elevation of protein tyrosine phosphatase activity in osteoclasts [42]. Genistein has a strong inhibitory effect on protein tyrosine kinases [15]. The inhibition of these kinases may induce apoptosis in a human ovarian tumor cell line and in Jurket T-leukemia cells [15]. Genistein may partly induce apoptosis of osteoclasts through a mechanism that inhibits protein tyrosine kinases in the cells. It has been shown that tyrosine kinase Src is implicated in the process of osteoclastinduced bone resorption in vitro and in vivo [47].

Culture with genistein has also been found to cause a significant increase in protein tyrosine phosphatase activity in osteoclasts [42]. This effect was also seen after the addition of genistein into the enzyme reaction mixture in vitro [42]. Genistein can directly activate protein tyrosine phosphatase in osteoclasts. Vanadate is 
an inhibitor of protein tyrosine phosphatase [48]. Culture of osteoclasts in the presence of vanadate did not cause a decrease in the number of osteoclasts. The suppressive effect of genistein on osteoclasts was also seen in the presence of vanadate [42]. It has been known that protein tyrosine phosphatase (Src homology 2 domain-containing tyrosine phosphatase) is a negative regulator of osteoclastogenesis and osteoclast-resorbing activity in mutant mice [49]. The suppressive effect of genistein on mature osteoclasts may partly be mediated through the activation of protein tyrosine phosphatase in the cells.

$\beta$-Glucuronidase is a lysomal enzyme, which is involved in the stimulation of bone resorption by PTH. Genistein did not have an effect on $\beta$-glucuronidase activity in osteoclasts [42]. The suppressive effects of genistein on osteoclastic bone resorption may not be implicated in the activity of lysosomal enzymes in the cells.

Thus, the suppressive effects of genistein on rat bone osteoclasts may be involved in cell apoptosis mediated through the $\mathrm{Ca}^{2+}$ siganaling mechanism, inhibition of protein kinase, and activation of protein tyrosine phosphatase in the cells.

Genistein regulates gene expression in osteoclasts. Genistein has been shown to regulate the gene expression of proteins that are related to osteoclast function. Culture with genistein $\left(10^{-6} \mathrm{M}\right)$ caused an increase in DNA fragmentation in isolated osteoclastic cells [50], indicating that genistein stimulates apoptotic cell death. In addition, the genistein-induced cell death was significantly prevented in the presence of caspase-3 inhibitor [50]. However, this effect was partial. Apoptotic cell death with genistein is partly mediated through activation of caspase-3, and also other mechanisms may participate in apoptotic cell death. The stimulatory effect of genistein $\left(10^{-6} \mathrm{M}\right)$ on the caspase- 3 mRNA expression was completely suppressed after culture with cycloheximide, an inhibitor of protein synthesis, or DRB, an inhibitor of transcriptional activity, in osteoclastic cells with or without macrophage colony stimulating factor (M-CSF) and RANKL [50]. This finding suggests that the effect of genistein on caspase- 3 mRNA expression is involved in protein synthesis and transcription activation in osteoclastic cells.

The RANK activation by binding of RANKL on preosteoclasts stimulates the $\mathrm{NF}-x \mathrm{~B}$ signaling, propbably through association with TRAF-6 in osteoclasts [51,52]. RANKL signaling targets include mitogen-activated protein kinases (MAPKs), NF- $x \mathrm{~B}$, and nuclear factor of activated T cells c1 (NFATc1) in osteoclast differentiation $[51,52]$. Culture with genistein $\left(10^{-6} \mathrm{M}\right)$ did not cause a significant change in NF- $x \mathrm{~B}$ mRNA expression in osteoclastic cells in the presence or absence of M-CSF and RANKL [44]. NFATc1 mRNA expression was significantly increased after culture with genistein $\left(10^{-6} \mathrm{M}\right)$ in osteoclastic cells cultured in the presence of M-CSF and RANKL [50]. Genistein may affect on the gene expression of NFATc1 that is a target protein of RANKL signaling in osteoclastic cells. The decrease in NFATc1 expression with the combination of genistein may be important in the revelation of the suppressive effect on osteoclastogenesis and osteoclastic cell function.

The activation of TRACP or cathepsin $\mathrm{K}$ plays a role in the promotion of osteoclastic bone resorption by stimulating the decomposition of bone matrix [50]. Genistein $\left(10^{-6} \mathrm{M}\right)$ did not have a significant effect on TRACP mRNA expression in osteoclastic cells [50]. Culture with genistein was found to induce a significant decrease in cathepsin $\mathrm{K}$ mRNA expression in osteoclastic cells cultured in the presence or absence of M-CSF and RANKL [50]. This finding demonstrates that genistein $\left(10^{-6} \mathrm{M}\right)$ has a suppressive effect on the activity of cathepsin $\mathrm{K}$ in osteoclastic cells, which are activated through RANKL signaling. Genistein has a potent suppressive effect on bone-resorbing activity.

\section{Intake of dietary soybean genistein prevents osteoporosis in animal model}

Bone mass decreases with age. Osteoporosis with a decrease in bone mass is widely recognized as a major public health problem. This decrease may be due to increased osteoclastic bone resorption and to decreased osteoblastic bone formation. Soybean genistein has been found to stimulate osteoblastic bone formation and inhibit osteoclastic bone resorption as shown in Figure 1. Whether the intake of dietary soybean genistein can reveal an anabolic effect on bone in vivo has been examined.

Nijiru, which is a by-product in the fermentation process of soybean to make fermented soybean (natto), contains large quantities of natural isoflavone [53]. Nijiru may be a functional food factor as a dietary isoflavone. The oral administration of the isoflavonecontaining soybean extract (Nijiru) to rats caused a significant increase in bone components (alkaline phosphatase activity, DNA and calcium contents) in the femoral-diaphyseal and -metaphyseal tissues in vivo [53], indicating the extract has an anabolic effect on bone metabolism. Also, Nijiru largely contains soybean saponin [54]. In addition, saponin has been shown to have a significant anabolic effect on bone components in the femoral tissues of rats [54].

Ovarian hormone deficiency at menopause stimulates bone loss. Ovariectomy (OVX) causes a lack of estrogen, and the hormone deficiency induces osteoporosis in humans [1-3]. Bone weight, bone mineral density, and bone mineral content were reduced in OVX rats [55]. The prolonged intake of fermented soybean diets with supplementation of isoflavone and saponin has been demonstrated to prevent bone loss in OVX rats [55], indicating that OVX-induced bone loss is prevented after the prolonged intake of dietary isoflavone and saponin supplementation.

The fermented soybeans are food sources that largely contain genistein. The effect of experimental diets using the fermented soybeans containing genistein on OVX-induced bone loss has also been demonstrated [56]. Experimental diets containing 44.6 to $92.4 \mathrm{mg}$ of isoflavones (including genistein, genistein, daidzein, and daidzein) per $100 \mathrm{~g}$ of diet was fed to OVX rats for 3 months. OVX caused a significant reduction in the dry weight, mineral density, calcium content, and alkaline phosphatase activity in the femoral tissues [56]. These reductions were prevented after feeding a natto diet [56]. Thus, the prolonged intake of dietary natto supplemented with isoflavone has a preventive effect on OVXinduced bone loss, suggesting that it may have a role in the prevention of osteoporosis.

The effect of a standardized soy extract (SSE, Soyselect) in the OVX rat model of menopause has also been examined [57]. OVX rats were orally treated for 6 weeks with the soy extract (50 or 100 $\mathrm{mg} / \mathrm{kg} /$ day), vehicle (distilled water), or $17 \beta$-estradiol $(0.5 \mathrm{mg} /$ $\mathrm{kg} /$ day) [57]. The SSE produced a bone-sparing effect associated with a slowing down in the increased bone turnover observed after OVX (as indicated by measurements of serum osteocalcin levels and excretion ratio of deoxypyridinoline) [57]. Remarkably, organ weight data and histopathologic analysis did not show any 
Figure 1: Role of genistein in bone homeostasis. Genistein stimulates osteoblastic bone formation and suppresses osteoclastic bone resorption, thereby increasing bone mass. Genistein stimulates protein synthesis and proliferation of osteoblastic cells, and it suppresses osteoclasogenesis from bone marrow stem cells and induces apoptosis of mature osteoclasts. Genistein regulates bone homeostasis through independent and dependent mechanisms on estrogen receptors. Genistein regulates the gene expression of many proteins that are related to osteoblastic bone formation and osteoclastic bone resorption.

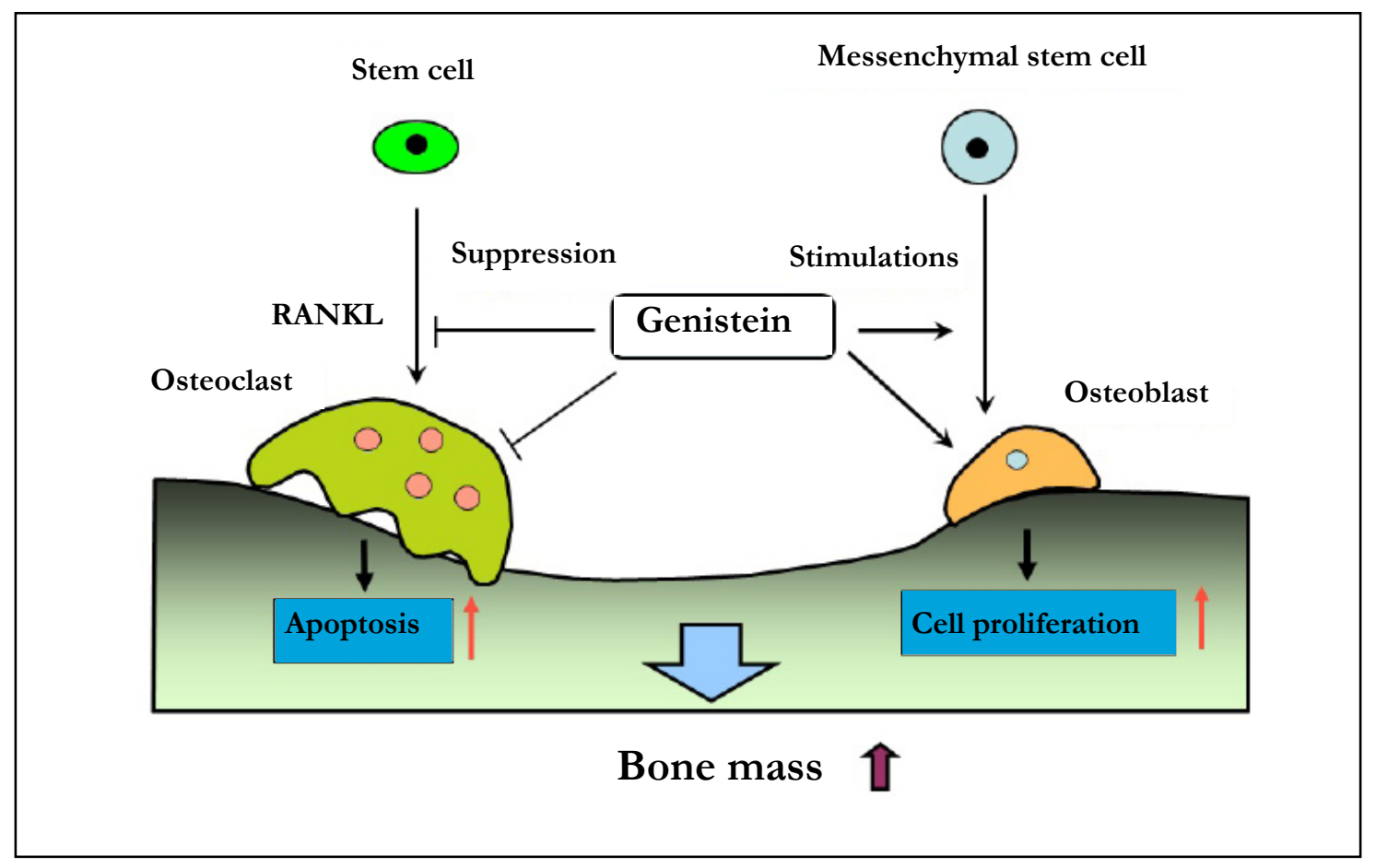

stimulatory activity of the SSE on the uterus [57]. Immunohistochemical analysis showed a significant down-regulation of estrogen receptor- $\alpha$ in uterine epithelium after $17 \beta$-estradiol treatment, but not after treatment with the SSE [57]. After treatment with $17 \beta$-estradiol, estrogen receptor- $\beta$ expression was not modulated in the stroma or epithelium, whereas the SSE induced an up-regulation of estrogen receptor- $\beta$ stromal expression [57]. Collectively, these findings suggest that the lack of stimulatory activity on the uterine epithelium using soy treatment could be due to a negligible stimulatory activity on estrogen receptor- $\alpha$ and/or to the enhanced expression observed in stromal estrogen receptor- $\beta$, the latter being considered as a negative modulator of estrogen receptor- $\alpha$-mediated uterine proliferation. Uterine epithelial progesterone receptor was down-regulated by $17 \beta$-estradiol, but not the SSE, as compared with that in OVX rats [57]. The minor increase in lipid levels induced by OVX was not affected by SSE administration [57]. Thus, it has been demonstrated that the tested extract has an interesting profile of tissue-specific response, in that it is efficacious in preventing experimental osteoporosis without causing stimulation in uterus at doses that are effective in bone.

\section{Preventive effect of dietary soybean genistein on osteoporosis in human subjects}

Twelve volunteers (six men and six women) were received Nijiru twice a day for 60 days at a dose of $1500 \mathrm{mg}$ (6 tablets) per day [58]. The serum $\gamma$-carboxylated osteocalcin concentration, which is a marker of osteoblastic bone formation, was significantly increased after the intake of Nijiru in both men and women to about 2 -fold that in the control group [58]. The intake of isoflavoneand saponin-containing Nijiru stimulated the $\gamma$-carboxylation of osteocalcin, which plays an important role in osteoblastic bone formation and mineralization, in healthy individuals [58]. The dietary intake of foods including genistein may have a role in the prevention of osteoporosis in human subjects.

Change in circulating biochemical markers of bone metabolism in aged individuals with the intake of fermented soybean (natto), which was made from isoflavone-rich soybean, has been examined [59]. Sixty-three volunteers (31 men and 32 women) were divided into four groups of 15 or 16 male volunteers and 16 or 16 female volunteers, and each group was sequentially given natto (40 g pack) containing once a day for 4 or 8 weeks as follows: either regular natto with naturally occuring isoflavone $35.0 \mathrm{mg}$ and calcium $51.4 \mathrm{mg}$ or supplemented natto containing isoflavone $35.0 \mathrm{mg}$ and calcium $60.0 \mathrm{mg}$.

As serum bone markers, bone-specific alkaline phosphatase, $\gamma$-carboxylated osteocalcin, bone tartrate-resistant acid phosphatase (TRACP), and N-telopeptide of type I collagen were assayed. The intake of regular natto for 4 or 8 weeks in men or women persons caused a significant increase in $\gamma$-carboxylated osteocalcin, a marker of bone formation, and a significant decrease in serum bone $\mathrm{N}$-teleopeptide of type I collagen, a marker of bone resorption, as compared with the value before intake [59]. Moreover, the intake of natto for 8 weeks in men or women caused a significant increase in serum bone-specific alkaline phosphatase activity and $\gamma$-carboxylated osteocalcin concentration and a significant decrease in serum bone TRACP activity and N-telopeptide of type I collagen, as compared with the values with the intake of regular natto [59]. This study suggests that the intake of regular natto with isoflavone-rich soybean has a stimulatory effect on bone formation and the suppressive effect on bone resorption in aged individuals. 
The prolonged intake of dietary genistein may be usefulness for bone health and the prevention of bone loss with aging and various pathophysiolocal states.

\section{Conclusion}

Bone homeostasis, which is regulated through osteoblastic cells and osteoclastic cells in bone tissues, is disordered with aging and pathophysiologic state including menopause. This leads to bone loss, which may be due to decreased osteoblastic bone formation and increased osteoclastic bone resorption. Bone loss induces bone fracture, which is a public problem. Functional food factors may help to prevent bone loss with aging. This may be important in maintaining bone health in long life. Genistein has been demonstrated to stimulate osteoblastic bone formation and to suppress osteoclastic bone resorption. The fermented soybeans (natto) are good food sources that largely contain genistein. The dietary fermented soybean intake has a preventive effect in the prevention and treatment of bone loss in animal models and humans. Moreover, the prevention of bone fracture with dietary fermented soybean intake is supported through epidemiologic investigation in Japan. Interestingly, soybean richly contains zinc, an essential trace element, which reveals a stimulative effect on bone formation and a suppressive effect on bone resorption [6]. Combination with genistein and zinc has been shown to reveal a synergistic-anabolic effect on bone mass [60]. The composition of genistein and zinc, which is named as Fosteum, has been developed as a novel supplement. This biomedical food factor has been demonstrated to be usefulness in the prevention and treatment of osteoporosis in human subjects.

\section{Disclosures}

All authors have no conflicts of interest.

\section{Acknowledgement}

The author (MY) was awarded from Mishima Kaiun Memorial Foundation, Japan, and Senji Miyata Foundation, Japan.

\section{References}

[1]. Cooper C, Melton J III (1995) Epidemiology of osteoporosis. Trends Endocrinol Metab 3: 224-229

[2]. Riggs B L, Jowsey J, Kelly PJ, Jones JD, Maher FT (1969) Effect of sex hormones on bone in primary osteoporosis. J Clin Invest 48: 1065-1072

[3]. Weitzmann MN, Pacifici R (2006) Estrogen deficiency and bone loss: an inflammatory tale. J Clin Invest 116: 1186-1194

[4]. Bonjour J-P, Schurch M-A, Rizzori R (1996) Nutritional aspects of hip fracture. Bone 18: 1395-1445

[5]. Yamaguchi M (2010) Nutritional Factors and Osteoporosis Prevention. Nova Science Publishers, Inc., New York, USA

[6]. Yamaguchi M (2010) Role of nutritional zinc in the prevention of osteoporosis. Mol Cell Biochem 338: 241-254

[7]. Yamaguchi M (2012) Role of beta-cryptoxanthin in bone homeostasis. J Biomed Sci 19:36-48

[8]. Parfitt AM (1990) Bone-forming cells in clinical conditions. In: Hall BK, editor. Bone Volume 1. The Osteoblast and Osteocyte. Boca Raton, FL: Telford Press and CRC Press, pp351-429

[9]. Baron R, Vignery A, Horowitz M (1984) Lymphocytes, macrophages and the regulation of bone remodeling. Bone Miner Res 2:175-243

[10]. Zaidi M, Blair HC, Moonga BS, Abe E, Huang C L-H (2003) Osteoclastogenesis, bone resorption, and osteoblast-based therapeutics. J Bone Miner Res 18: 599-609

[11]. Chambers TJ, Fuller K (2011) How are osteoclasts induced to resorb bone Ann N Y Acad Sci 1240:1-6

[12]. Canalis E, McCarthy T, Centrella M (1988) Growth factors and the regulation of bone remodeling. J Clin Invest 81: 277-281

[13]. Raggatt LJ, Partridge C (2010) Cellular and molecular mechanisms of bone remodeling. J Biol Chem 285:25103-25108

[14]. Chen G, Deng C, Li YP (2012) TGF- and BMP signaling in osteoblast differentiation and bone formation. Int J Biol Sci 8:272-288

[15]. Liu Y, Bhalla K, Hill C, Priest DG (1994) Evidence for involvement of tyrosine phosphorylation in taxol-induced apoptosis in a human ovarian tumor cell line. Biochem Pharmacol 48: 1265-1272

[16]. Yamaguchi M, Gao YH (1997) Anabolic effect of genistein on bone metabolism in the femoral-metaphyseal tissues of elderly rats is inhibited by the anti-estrogen tamoxifen. Res Exp Med 197: 101-107

[17]. Yamaguchi M, Gao YH (1998) Anabolic effect of genistein and genistein on bone metabolism in the femoral-metaphyseal tissues of elderly rats: The genistein effect is enhanced by zinc. Mol Cell Biochem 178: 377-382

[18]. Kuiper GG, Lemmen JG, Carlsson B, Corton JC, Safe SH, van der Saag PT, van der Burg B, Gustafsson JA (1998) Interaction of estrogenic chemicals and phytoestrogens with estrogen receptor beta. Endocrinology 139: $4252-$ 4263

[19]. Gao YH, Yamaguchi M (1999) Anabolic effect of daizein on cortical bone in tissue culture: Comparison with genistein effect. Mol Cell Biochem 194: 93-98

[20]. Yamaguchi M, Zhong JM, Fushimi T (2002) Anabolic effect of phosphogenistein and phosphodaidzein on bone components in rat femoral-metaphyseal tissues in vitro. J Bone Miner Metab 20: 148-155

[21]. Yamaguchi M, Ma ZJ (2001) Effect of polyphenols on calcium content and alkaline phosphatase activity in rat femoral tissues in vitro. Biol Pharm Bull 24: $1437-1430$

[22]. Sugimoto E, Yamaguchi M (2000) Anabolic effect of genistein is osteoblastic MC3T3-E1 cells. Int J Mol Med 5: 515-520

[23]. Sugimoto E, Yamaguchi M (2000) Stimulatory effect of daidzein in osteoblastic MC3T3-E1 cells. Biochem Pharmacol 59: 471-475

[24]. Yamaguchi M, Sugimoto E (2000) Stimulatory effect of genistein and daizein on protein synthesis in osteoblastic MC3T3-E1 cells: Activation of aminoacyl-tRNA synthetase. Mol Cell Biochem 214: 97-102

[25]. Yamaguchi M, Weitzmann MN. (2009) The estrogen 17-estradiol and phytoestrogen genistein mediate differential effects on osteoblastic NF-B activity. Int J Mol Med 23: 297-301

[26]. Uchiyama S, Yamaguchi M (2007) Genistein and zinc synergistically enhance gene expression and mineralization in osteoblastic MC3T3-E1 cells. Int J Mol Med 19: 213-220

[27]. Majeska RJ, Wuthier RE (1975) Studies on matrix vesicles Isolated from chick epiphyseal cartilage. Association of pyrophosphatase and ATPase activities with alkaline phosphatase. Biochim Biophys Acta 391: 51-60

[28]. Lian JB, Stein GS, Cannalis E, Roky PG, Boskey AL (1999) Bone formation: Osteoblast lineage cells, growth factor, matrix protein, and the mineralization process. In: Favus MJ (Ed), Primer on the Metabolic Bone Disease and Disorders of Mineral Metabolism (4th edition), pp14-29, New York, Lippincott Williams \& Wilkins Press

[29]. Pan W, Quarles LD, Song LH, Yu YH, Jiao C, Tang HB, Jiang CH, Deng HW, Li YJ, Zhou HH, Xiao ZS (2005) Genistein stimulates the osteoblastic differentiation via N0/cGMP in bone marrow culture. J Cell Biochem 94:307-316

[30]. Tang X, Zhu X, Liu S, Wang S, Ni X (2011) Isoflavones suppress cyclic adenosine 3',5'-monophosphate regulatory element-mediated trnscription in osteoblastic cell line. J Nutr Biochem 22:865-873

[31]. Klein-Nulend J, Fall PM, Raisz LG (1990) Comparison of the effects of synthetic human parathyroid hormone (PTH)-(1-34)-related peptide of malignancy and bovine PTH-(1-34) on bone formation and resorption in organ culture. Endocrinology 126: 223-227

[32]. Graves L III, Jilka R L (1990) Comparison of bone and parathyroid hormone as stimulators of osteoclast development and activity in calvarial cell cultures from normal and osteopetrotic (mi/mi) mice. J Cell Physiol 145: 102-109

[33]. Klein DC, Raisz LG (1970) Stimulation of bone resorption in tissue culture. Endocrinology 86: 1436-1440

[34]. Yamaguchi M, Gao YH (1998) Inhibitory effect of genistein on bone resorption in tissue culture. Biochem Pharmacol 55:71-76

[35]. Takahashi N, Yamana H, Yoshiki S, Roodman GD, Mundy GR, Jones SJ, Boyde A, Suda T (1988). Osteoclast-like cell formation and its regulation by osteotropic hormones in mouse bone marrow cultures. Endocrinology 122: $1373-1382$

[36]. Gao YH, Yamaguchi M (1999) Inhibitory effect of genistein on osteoclastlike cell formation in mouse marrow cultures. Biochem Pharmacol 58: $767-$ 772

[37]. Chen XW, Garner SC, Anderson JJ (2002) Isoflavones regulate interleukin- 6 and osteoprotegerin synthesis duering osteoblast cell differentiation via an estrogen-receptor-dependent pathway. Biochem Biophys Res Commun 295:417-422

[38]. Viereck V, Grundker C, Blaschke S, Siggelkow H, Emons G, Hofbauer LC (2002) Phytoestrogen genistein stimulates the production of osteoprotegerin in human trabecular osteoblasts. J Cell Biochem 84:725-735

[39]. Garcia Palacios V, Robinson LJ, Borysenko CW, Lehmann T, Kalla SE, Blair 
HC (2005) Negative regulation of RANKL-induced osteoclastic differentiation in RAW264.7 cells by estrogen and phytoestrogens. J Biol Chem 280:13720-13727

[40]. Karieb S, Fox SW (2011) Phtoestrogens directly inhibit TNF- $\bigotimes$-induced bone resorption in RAW264.7 cells by suppressing c-fos-induced NFATc1 expression. J Cell Biochem 112:476-487

[41]. Gao YH, Yamaguchi M (1999) Suppressive effect of genistein on rat bone osteoclasts: Apoptosis is induced through $\mathrm{Ca} 2+$ signaling. Biol Pharm Bull 22: 805-809

[42]. Gao YH, Yamaguchi M (2000) Suppressive effect of genistein on rat bone osteoclasts: Involvement of protein kinase inhibition and protein tyrosine phosphatase activation. Int J Mol Med 5: 261-267

[43]. Murrills RJ, Shane E, Lindsay R, Dempster DW (1989)Bone resorption by isolated human osteoclasts in vitro: effects of calcitonin. J Bone Miner Res 4: 259-268

[44]. Su Y, Charkrabort M, Nathanson MH, Baron R (1992) Differential effects of the 3',5'-cyclic adenosine monophosphate and proein kinase $\mathrm{C}$ pathways on the response of isolated rat osteoclasts to calcitonin. Endocrinology 131: 1497-1502

[45]. Jones DOP, McConkey DJ, Nicotera P, Orrenius S (1989) Calcium-activated DNA fragmentation in rat liver nuclei. J Biol Chem 264: 6398-6403

[46]. McCabe MJ, Orrenius S (1993) Genistein induces apoptosis in immature human thymocytes by inhibiting topoisomerase-II. Biochem Biophys Res Commun 194: 944-950

[47]. Missbach M, Jeschke M, Feyen J, Muller K, Glatt M, Green J, Susa M (1999) A novel inhibitor of the tyrosine kinase Src suppresses phosphoryalation of its major cellular substrates and reduces bone resorption in vitro and in rodent models in vivo. Bone 24: 437-449

[48]. Hunter T (1995) Protein kinases and phosphatases: the Yin and Yang of protein phosphorylation and signaling. Cell 80: 225-236

[49]. Aoki K, Didomenico E, Sims NA, Mukhopadhyay K, Neff L, Houghton A, Amling M, Levy JB, Horne WC, BaronR (1999) The tyrosine phosphatase SHP-1 is a negative regulator of osteoclastogenesis and osteoclast resorbing activity: increased resorption and osteopenia in mev.mev mutant mice. Bone 25: 261-267

[50]. Uchiyama S, Yamaguchi M (2007) Genistein and zinc synergistically stimulate apoptotic cell death and suppress RANKL siganling-related gene expression in osteoclastic cells. J Cell Biochem 101: 529-542
[51]. Asagiri M, Takayanagi H (2007) The molecular understanding of osteoclast differentiation. Bone 40:251-264

[52]. Inoue J, Ishida T, Tsukamoto N, Kobayashi N, Naito A, Azuma S, Yamamoto T (2000). Tumor necrosis factor receptor-associated factor (TRAF) family: adaptor proteins that mediate cytokine signaling. Exp Cell Res 254:14-24

[53]. Ono R, Yamaguchi M (1999) Increase in bone components of rats orally administered isoflavone-containing soybean extract (Nijiru). J Health Sci 45: 66-69

[54]. Ono R, Yamaguchi M (1999) Anabolic effect of soybean saponin on bone components in the femoral tissues of rats. J Health Sci 45: 251-255

[55]. Ono R, Ma ZJ, Yamaguchi M (2000) Prolonged intake of fermented soybean diets with supplementation of isoflavone and saponin prevents bone loss in ovariectomized rats. J Health Sci 46: $70-74$

[56]. Ma ZJ, Shimanuki S, Igarashi A, Kawasaki Y, Yamaguchi M (2000) Preventiv effect of dietary fermented soybean on bone loss in ovariectomized rats: Enhancement with isoflavone and zinc supplementation. J Health Sci 46: 263-268

[57]. Gallo D, Zannoni GF, Apollon P, Martinelli E, Ferlin C, Passetti G, Riva A, Morazzoni P, Bombardelli E, Scambia G (2005) Characterization of the pharmacologic profile rat model of menopause: effects on bone, uterus, and lipid profile. Menopause 12:589-600

[58]. Yamaguchi M, Ono R, Ma ZJ (2001) Prolonged intake of isoflavone- and saponin-containing soybean extract (Nijiru) supplement enhances circulating -carboxylated osteocalcin concentration in healthy individuals. J Health Sci 47: 579-58239

[59]. Yamaguchi M, Igarashi A, Sakai M, Degawa H, Ozawa Y (2005) Prolonged intake of dietary fermented isoflavone-rich soybean reinforced with zinc affects circulating bone biochemical markers in aged individuals. J Health Sci 51: 191-196

[60]. Yamaguchi M (2012) Nutritional factors and bone homeostasis: Synergistic effect of zinc and genistein osteogenesis. Mol Cell Biochem 366:201-221 